\title{
Le choix entre les débits moyens mensuels, décadaires et journaliers pour les projets d'utilisation des ressources en eau
}

PAR

\author{
R. André et J.-R. Tiercelin* \\ Division Hydrologie.Hydranlique Fluviale \\ Centre Technique du Génie Rural des Eaux et Forêts (C'TREF) Antony
}

L'auteur d'un projet faisant intervenir la prise en compte de ressources provenant d'un cours d'eau doit choisir le pas de temps auquel il étudiera les ressources qui l'intéressent.

Ce choix devrait être un compromis entre :

- la recherche de la simplification qui suggère d'adopter un long pas de temps, car la complexité des méthodes hydrologiques croit rapidement lorsque le pas de temps décroît ;

- la recherche de la meilleure précision possible dans le projet, qui suggère au contraire d'adopter un pas de temps court.

L'expérience montre que le premier terme de l'alternative prédomine largement en général. Notre propos est donc de mettre en garde le projeteur contre les simplifications abusives, et de l'aider dans le choix de l'échelle de temps qui paraît la mieux adaptée lorsqu'il entreprend son travail.

\section{Pas de temps étudiés}

Nous n'envisagerons qu'un nombre limité de pas de temps possibles, les autres ayant un intérêt pratique bien moindre. Les échelles de temps supérieures au mois n'ont d'intérêt que pour des études très schématiques, et perdent leur intérêt, à notre avis, en raison du développement du calcul automatique. A l'autre extrème, les pas de temps inférieurs à la journée n'ont d'intérêt que pour les problèmes de crues, qui sortent de notre propos.

(*) Actuellement à la Direction Départementale de l'Agriculture de la Réunion.
L'échelle de temps mensuelle est d'une pratique très courante et son utilisation est bien connue. Elle se prête bien à la description de systèmes complexes faisant intervenir simultanément plusieurs séries de variables hydrologiques, et la procédure de simulation des débits mensuels est simple (on trouvera une bonne synthèse de la question en langue française dans Dauty J. - "Les débits mensuels. Simulation et exploitation pour la gestion des ressources" Thèse (1972). Université des Sciences \& Techniques du Languedoc. Montpellier)

L'échelle de temps décadaire, dont l'utilisation se répand actuellement est justiciable des mêmes procédés mathématiques que l'échelle de temps mensuelle; elle ne constitue donc pas une complication notable par rapport à cette dernière, il y a simplement une quantité importante de données à manipuler.

L'échelle de temps journalière enfin, n'est utilisable qu'en recourant à des modèles de mise au point et mise en ouvre assez complexes, mais doit apporter la meilleure précision dans certains types de problèmes, et est susceptible de se prêter à la simulation.

Les échelles de temps intermédiaires entre les 3 échelles de temps ci-dessus ne semblent pas a priori devoir prsenter beaucoup d'intérêt pratique, et leur prise en compte ne ferait qu'alourdir l'exposé.

\section{Définition d'exemples-types d'aménage- ments}

Le choix à faire est une question de cas d'espèce. Il est évidemment impossible de faire le tour de tous les types de projets possibles; nous considérons simplement quelques 
cas très simples auxquels peuvent s'apparenter bon nombre de projets, tout au moins sur le plan de l'échelle de temps la mieux adaptée.

Dans les trois cas exposés, on suppose qu'il s'agit de satisfaire des besoins constants toute l'année en prélevant de l'eau en un point unique d'une rivière, constitué par une réserve située sur le cours même de cette rivière. Les 3 cas considérés correspondent à un ordre de grandeur différent du volume de la réserve par rapport aux apports en eau :

- prélèvement au fil de l'eau (réserve nulle)

-n régularisation saisonnière

- régularisation interannuelle.

Il n'y a évidemment pas de limite stricte entre les deux derniers cas, et nous prendrons soin d'adopter des exemples suffisamment tranchés pour qu'il n'y ait pas d'ambiguité.

Dans les exemples simplifiés que nous utilisons, nous supposons que les réserves sont gérées de la façon la plus simple qui se puisse concevoir : on satisfait en totalité les demandes tant que cela est possible, et on emmagasine systématiquement les excédents éventuels dans la réserve tant que cette dernière n'est pas pleine.

\section{Définition des défaillances}

En considérant un tel système, ou n'importe quel autre plus complexe, le projeteur se posera la question de savoir dans quelle mesure il répond à son problème concret, ou au contraire quels sont les risques de défaillance du système vis à vis des besoins.

Suivant les cas d'application, une défaillance pourra être définie par sa durée, par son volume, ou par toute autre caractéristique plus complexe. Pour limiter le nombre de cas étudiés, nous définirons ici une défaillance par son volume total sur une durée de un an.

Pour le type de problème qui nous intéresse, il est assez intuitif que les résultats doivent fortement dépendre de l'tmportance relative des grandeurs en jeu : volume des apports, volume de la demande et volume de la réserve.

Pour que les démonstrations aient la plus grande généralité possible, nous avons raisonné sur des variables calées par rapport à certaines caracteristiques des stations utilisées. Pour ce qui est du débit demandé, supposé constant au cours de l'année, on considère le module $M$ à la station, un débit $m$ caractéristique des étiages (choisi pour êtic d'un calcul automatique facile et d'une bonne stabilité statistique), et le débit demandé est pris égal à une des valeurs $m ; m+0,1(M-m) ; m+0,2(M-m) ; \ldots M$. Le dernier cas correspond donc à l'utilisation de la totalité des ressources en eau du cours d'eau d'une année moyenne.

Quant à la réserve, elle est exprimée en pourcentage de l'apport moyen annuel de la rivière. Ceci facilite les comparaisons entre divers aménagements et correspond par ailleurs à une pratique fréquente, en particulier toutes les fois où les besoins ne sont pas encore bien définis.

Enfin, les volumes de défaillance sont exprimés en pourcentage de la demande annuelle, mais aussi en pourcentage de l'apport moyen annuel, ceci pour faciliter les comparaisons entre les différents cas.

\section{Choix des cours d'eau étudiés}

Par ailleurs, les résultats sont susceptibles d'être fortement influencés par le régime de la rivière, le substratum et la superficie du bassin versant concerné, qui conditionnent l'irrégularité des débits. Bien que les corrections d'échelle adoptées soient susceptibles d'atténuer ces différences, nous avons considéré simplement:

- un bassin à régime pluvial sur substratum perméable : la Seine à Bar ;

-..- un bassin à régime pluvio-nival sur substratum varié : le Salat à Kercabanac ;

- un bassin à régime pluvial sur substratum imperméable: le Cousin à Cussy,

qui représentent les conditions rencontrées dans une importante proportion du territoire français.

\section{Résultats de l'étude de défaillance}

Les figures ci-après représentent des études de probabilité de défaillance dans 9 cas, resultant de la combinaison de trois types de problèmes (aménagement au fil de l'eau, régularisation saisonnière, régularisation inter-annuelle) et des trois rivières étudiées.

Dans chaque graphique sont comparés les résultats obtenus en considérant les débits comme une succession de moyennes mensuelles, de moyennes décadaires ou de moyennes journalieres.

Pour la lecture des graphiques, on verra par exemple que s'il s'agit d'un prélèvement au fil de l'eau à Bar, et que l'on considère le pas de temps mensuel, il y a dans $25 \%$ des cas dépassement d'une défaillance supérieure à $10 \%$ de la demande annuelle.

La longueur des séries d'observations était parfois très courte, il y a une forte irrégularité dans les graphiques, et la différence des résultats obtenus avec les trois échelles de temps ne peut être valablement appréciée qu'en considérant l'ensemble d'un graphique, et non pas des valeurs isolées.

Cette irrégularité sera d'ailleurs souvent atténuée dans la pratique en utilisant de très longues séries de données obtenues par simulation, mais pour la présente démonstration nous avons préféré utiliser les données brutes, car l'usage de la simulation aurait introduit des biais susceptibles de diminuer la crédibilité de la démonstration.

Bien entendu, les cas proposés dans les graphiques représentent un échantillon infinitésimal des circonstances pratiques que l'on rencontre, ou que l'on peut imaginer, mais couvrent néanmoins un large éventail dans l'ordre de grandeur relatif des quantités en jeu : prélèvements, apports et réserves. Ils peuvent donc constituer un repère utilisable dans le choix du pas de temps adapté à un problème concret. 


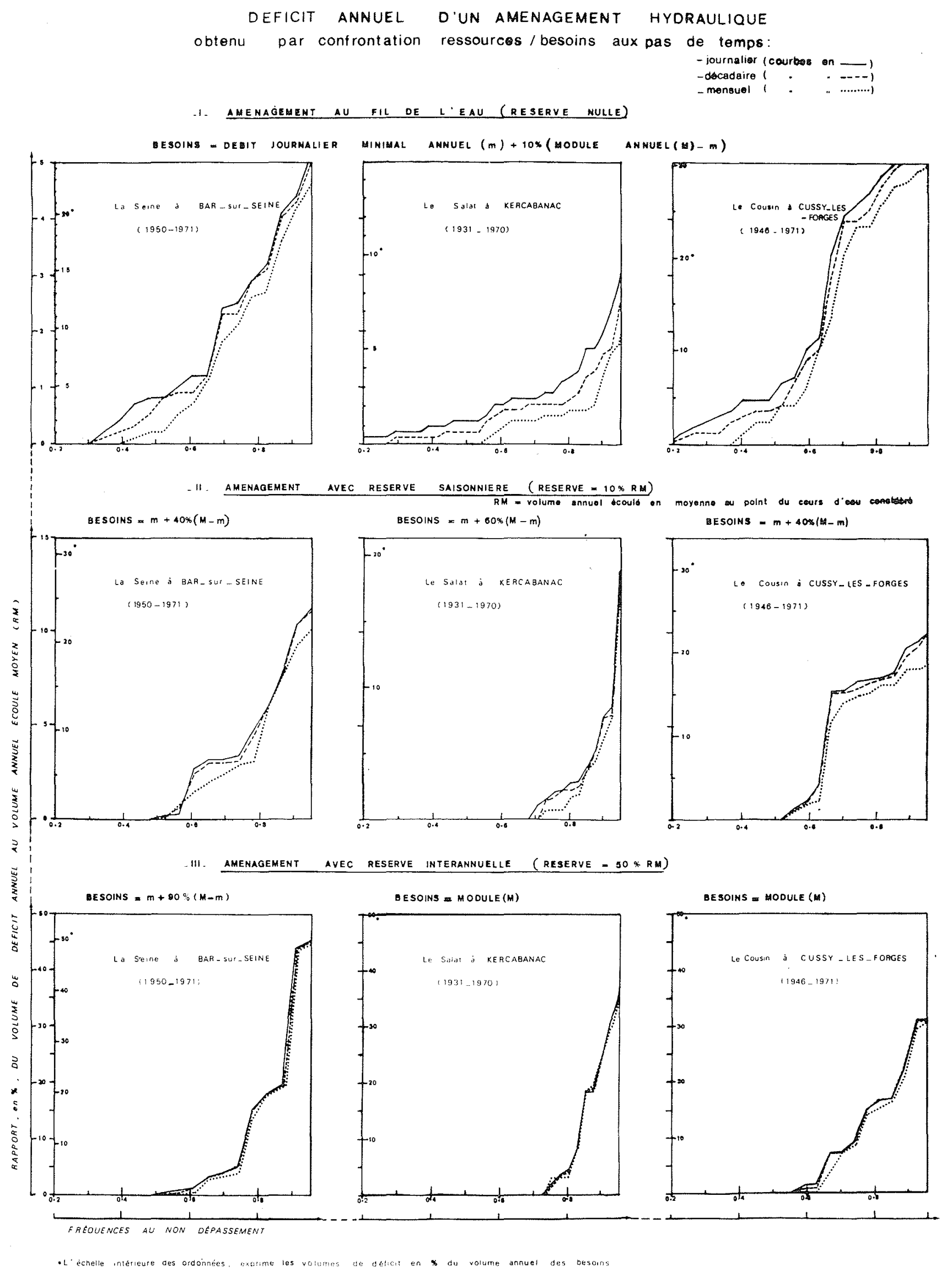




\section{Commentaires sur les résultats}

Les éléments d'ordre hydrologique ou hydraulique n'entrent pas seuls en ligne de compte dans la conception d'un système hydraulique, mais souvent également des éléments d'ordre économique, de sorte que si une incertitude considérable plane sur ces derniers, il est inutile de rechercher une trop grande précision dans la prise en compte des premiers.

En définitive, il n'est donc pas possible de conclure par une recette générale ; chaque cas concret doit faire l'objet d'une appréciation particulière, mais nous pensons que des points de repère du type de ceux que nous avons présentés peuvent aider au choix du pas de temps convenable. Le projeteur aura d'ailleurs dans la mesure du possible intérêt à construire un graphique de ce type, pour les rivières qu'il étudie dans son projet, à partir de quelques années d'observations, pour apprécier l'intérêt qui peut s'attacher à mettre en oeuvre un pas de temps court et un outil hydrologique relativement complexe et couteux ; ce travail exploratoire peut être exécuté à la main clans les cas simples.

Quelques conclusions peuvent être néanmoins tirées des graphiques que nous avons présentés :

- d'une façon générale : la simplification de la chronique des débits conduit à une appréciation optimiste des risques de défaillance d'un système :
- pour les aménagements au fil de l'eau (ou avec une faible réserve) : la différence entre pas de temps journaliers, décadaires et mensuels peut être importante, de sorte que l'usage du pas de temps joumalier sera souvent justifié ;

- pour les aménagements à réserve saisonnière : la différence entre pas de temps mensuel et décadaire peut être telle que les débits moyens décadaires semblent souvent devoir être retenus, d'autant plus que, comme nous l'avons vu, leur structure mathématique n'est pas plus complexe que celle des debits moyens mensuels; quant à l'écart entre pas de temps décadaire et pas de temps journalier, il n'est pas forcément négligeable ;

- pour les aménagements à réserve interannuelle : les débits journaliers ne présentent pas d'intérêt. L'usage du pas de temps décadaire ne doit pas être forcément exclu, mais les débits moyens mensuels semblent devoir suffire dans la plupart des cas.

Les indications générales ci-dessus devront être adaptées à chaque cas concret particulier, mais de toute façon l'essentiel pour le projeteur d'un aménagement hydraulique est de prendre conscience que, pour simplifier la tâche, ou en raison d'idées reçues qui proviemnent d'une époque encore proche où le calcul automatique et les outils hydrologiques n'étaient pas ce qu'ils sont actuellement, il risque d'apprécier de façon exagérément optimiste la garantie de service qu'est susceptible d'apporter le projet qu'il propose. 